\title{
Convex, Monotone Systems are Optimally Operated at Steady-State*
}

\author{
Marius Schmitt ${ }^{1}$, Chithrupa Ramesh ${ }^{1}$, Paul Goulart ${ }^{2}$ and John Lygeros ${ }^{1}$
}

\begin{abstract}
We consider a special class of monotone systems for which the system equations are also convex in both the state and the input. For such systems we study optimal infinite horizon operation with respect to an objective function that is also monotone and convex. The main results state that, under some technical assumptions, these systems are optimally operated at steady state, i.e. there does not exist any timevarying trajectory over an infinite horizon that outperforms stabilizing the system in the optimal equilibrium. We draw a connection to recent results on dissipative systems in the context of Economic Model Predictive Control, where systems that are optimally operated at steady state have already been studied. Finally, we apply the main result to a problem in traffic control, where we are able to disprove the existence of improving periodic trajectories involving the alternation of congestion and free flow for freeway ramp metering.
\end{abstract}

\section{INTRODUCTION}

In this work we consider a special class of nonlinear, monotone systems. Monotone systems are systems for which trajectories preserve a partial ordering on the states [1], [2], [3], a fact that is favorable for controller design and has recently been exploited in a series of papers [4], [5], [6]. Even stronger results can be obtained for positive systems, that is, systems that are not just monotone, but also linear [7], [8], [9]. Here, we consider the more general class of nonlinear, state-monotone systems, whose system equations are jointly convex in state and input. For so-called convexmonotone systems [10], certain control tasks which are difficult for general, nonlinear systems are in fact tractable. Most significantly, it has been shown that the state of convex, monotone systems (at any time) is given as a convex function of the control inputs, which makes certain optimization problems over trajectories tractable [10].

We study the problem of optimal average, infinite horizon control of convex, state-monotone systems with respect to a convex, state-monotone cost, in the absence of any noise or disturbances and assuming perfect knowledge of state and system dynamics. For controllable, linear systems and radially unbounded, convex objectives, such a trajectory planning problem is not very challenging since there exists an optimal (minimal cost) equilibrium and any controller that stabilizes the system to this equilibrium achieves optimal average, infinite horizon cost. However, this question is not as simple for general, nonlinear systems. In particular, the

*Research was supported by the European Union 7th Framework Program "Scalable Proactive Event-Driven Decision-making (SPEEDD)" (FP7-ICT 619435)

${ }^{1}$ Automatic Control Laboratory, ETH Zurich, Switzerland, <schmittm, rameshc, lygeros>dcontrol.ee. ethz.ch

${ }^{2}$ Department of Engineering Science, University of Oxford, UK, paul.goulart@eng.ox.ac.uk optimal operation of systems for which the costs represent actual, economic performance criteria like operation costs, production output or throughput of some commodity through a network, is studied in the context of Economic Model Predictive Control (MPC) [11], [12]. Depending on the system, the optimal operation might very well involve the system repeating a cycle [13], [14]. Therefore, the question of which systems are optimally operated at steady state, that is, systems for which there does not exist a periodic (or a nonperiodic) trajectory improving average infinite horizon cost over the cost incurred in the optimal equilibrium, is of importance. It has been shown that, under some technical assumptions, the class of systems that are optimally operated at steady-state is exactly the class of systems that are dissipative with respect to a particular storage function [15]. However, finding a certificate that a particular system is dissipative is difficult. The main result of this work is to show that convex, state-monotone systems are optimally operated at steady state.

We apply this theoretical result to the problem of freeway ramp metering. Ramp metering refers to the active control of the inflow of cars on a freeway via the onramps, by means of traffic lights [16], [17]. We consider a (quasi-) steadystate situation as in [18], in which the traffic demand cannot be completely served, a common occurrence during rushhour periods. Using our theoretical results, we can prove that for the commonly used monotone Cell Transmission Model (CTM) [19], [20], there does not exist a control policy that improves average demand satisfaction over the one achieved in the optimal equilibrium. This answers a question posed in [21] regarding the possibility to improve throughput by using time varying trajectories involving the alternation of congestion and free flow periods.

The problem description is formalized next in Section II. The theoretical results are derived in Section III. In Section IV, we apply the results to the ramp metering case and we provide concluding remarks in Section V.

We will use the following notation: all inequalities that involve vectors are to be understood component-wise. We use subscripts to index components of a vector or elements of a scalar-valued sequence, that is, $x_{k} \in \mathbb{R}$ can denote the $k^{\text {th }}$ component of a vector or the $k^{\text {th }}$ element of a sequence. We exclusively consider systems in discrete time $t \in \mathbb{Z}_{0}^{+}$ and for quantities that evolve over time, like the state of a system, we write $x(t)$. For a given discrete-time system and the input sequence $u=(u(0), u(1), \ldots, u(T-1))$ of finite or infinite length, we denote the system trajectory for the input $u$ starting at $x(0)$ as $x_{u}(t, x(0))$, if we want to emphasize that it is valid trajectory. If we are interested in the value 
at a particular time $t$, we also use shorthand notation $x(t)=$ $x_{u}(t, x(0))$. The average cost over any finite trajectory will be denoted $c\left(x_{u}(t, x(0))\right):=\frac{1}{T} \sum_{t=0}^{T-1} c(x(t), u(t))$.

\section{Problem statement}

Consider a nonlinear, discrete time dynamical system with state $x(t) \in \mathbb{X} \subseteq \mathbb{R}^{n}$ and input $u(t) \in \mathbb{U} \subseteq \mathbb{R}^{m}$ given as

$$
x(t+1)=f(x(t), u(t)) .
$$

with $f: \mathbb{X} \times \mathbb{U} \rightarrow \mathbb{X}$. In this work, we study systems with convex and monotone dynamics:

Definition 1: A system is state-monotone if the system equations $f: \mathbb{X} \times \mathbb{U} \rightarrow \mathbb{R}^{n}$ are component-wise nondecreasing in the state, i.e. in its first $n$ arguments. A system is convex if the system equations $f(x, u)$ are jointly convex in $x$ and $u$ and the sets $\mathbb{X}$ and $\mathbb{U}$ are closed and convex. We write convex, state-monotone (CSM) system for systems that satisfy both properties.

The standard definition of a monotone system as used in [1], [2], [3] is equivalent to the system equation $f$ being component-wise nondecreasing in all its arguments, whereas we only require monotonicity in the states. In that sense, our problem setup is a generalization of the notion of convexmonotone systems, as introduced recently in [10].

We will analyze the performance of controlling CSM systems with respect to the minimization of a lowersemicontinuous CSM cost $c: \mathbb{X} \times \mathbb{U} \rightarrow \mathbb{R} \cup\{+\infty\}$. The definition of a CSM cost is analogous to Definition 1, except that the cost may become infinite and therefore, we only require lower semicontinuity instead of continuity.

Assumption 1: We assume that sets $\mathbb{X}$ and $\mathbb{U}$ and the cost function $c$ satisfy the following conditions:

(i) The set $\mathbb{X}$ is contained in a translation of the positive cone, that is, there exists a finite $\underline{x} \in \mathbb{R}^{n}$ such that $\underline{x} \leq x$ for all $x \in \mathbb{X}$.

(ii) The set $\mathbb{X}$ is positively control invariant for all $u \in \mathbb{U}$.

(iii) The set $\mathbb{U}$ is compact.

(iv) The cost is unbounded in positive directions, that is, for every $c \geq 0$, there exists $\bar{x}$ such that for every $x: x \geq \bar{x}$, it follows that $c(x, u) \geq c(\forall u \in \mathbb{U})$.

(v) The minimum $c^{*}=\min _{x \in \mathbb{X}, u \in \mathbb{U}} c(x, u)$ s.t. $x=f(x, u)$ is attained.

Under assumption (v), there exists a (potentially nonunique) minimizer $\left(x^{*}, u^{*}\right)=\underset{x \in \mathbb{X}}{\operatorname{argmin}} c(x, u)$ s.t. $x=f(x, u)$ that we will call the optimal equilibrium. Conditions (i,ii,iii,iv) imply that there do not exist cost-optimal trajectories for which certain states diverge. In practice, divergence of the states is hardly ever a desired outcome ${ }^{1}$. These conditions can be relaxed in certain cases, for example, control invariance is not necessary if the system is exponentially stable and the cost on actuation is "sufficiently large". Also, conditions (i,iv) can be omitted if $\mathbb{X}$ is compact.

\footnotetext{
${ }^{1}$ Note that the assumption of radially unbounded state costs, which is often invoked to exclude such a behavior, is not suitable in this case, because of the assumption of monotonicity of the cost in the states.
}

In Section III, we show that the systems of interest are in fact dissipative with respect to a specific supply function and that this is a crucial property to explain optimal operation of the systems in infinite horizon. The literature on dissipative systems, e.g. [14], [12], provides different sets of criteria for determining when dissipativity of a system is equivalent to the system being optimally operated at steady state and parts of these criteria are interchangeable with the ones presented here.

\section{THEORY}

We seek to compare the long-term performance achievable by stabilizing the system at the optimal equilibrium with the minimal infinite horizon average cost of any feasible trajectory. To this end, we first need to show an intermediate result that will be helpful in constructing cost-efficient equilibria.

Lemma 1: Given a CSM system with CSM cost satisfying Assumption 1, assume there exists $x \in \mathbb{X}$ and $u \in \mathbb{U}$ such that $x \geq f(x, u)$. Then, there exists an equilibrium $x^{\prime}=f\left(x^{\prime}, u^{\prime}\right)$, $x^{\prime} \in \mathbb{X}, u^{\prime} \in \mathbb{U}$ with $c\left(x^{\prime}, u^{\prime}\right) \leq c(x, u)$.

Proof: Consider the fixed point iteration

$$
x(t+1)=f(x(t), u), \quad x(0):=x .
$$

From the inequality above, we know that $x(1)=f(x, u) \leq$ $x(0)=x$. Because of monotonicity of the system, we can conclude that $x(t+1) \leq x(t)$ inductively. The iterates $x(t)$ can be interpreted as the system trajectory $x_{u}(t, x(0))$ for constant control inputs $u \in \mathbb{U}$. By Assumption 1 , the set $\mathbb{X}$ is positively control invariant and any $x(t) \in \mathbb{X}$ is bounded below by $\underline{x} \leq$ $x(t)$. Boundedness and monotonicity of the sequence imply that the fixed point iteration converges to some $x_{\infty}=f\left(x_{\infty}, u\right)$. Note that $x_{\infty} \in \mathbb{X}$ because $\mathbb{X}$ is closed and furthermore, $x_{\infty} \leq x$ which implies, by monotonicity of the objective in the states, that $c\left(x_{\infty}, u\right) \leq c(x, u)$.

Using this intermediate result, we first restrict our attention to periodic trajectories. If a finite trajectory returns to the initial state, i.e. $x_{u}(T, x(0))=x(0)$, we call it a cycle. The following result shows how to construct improving equilibria from cycles.

Theorem 1: Let $f$ be a CSM system with $c$ a CSM cost satisfying Assumption 1. The average cost of any cycle $x_{u}(t, x(0))$ of finite, but arbitrary length $T$ is lower bounded by the cost in the optimal equilibrium.

Proof: For notational convenience, let us define the average state $\bar{x}:=\frac{1}{T} \sum_{t=0}^{T-1} x_{u}(t, x(0))$ and average input $\bar{u}:=$ $\frac{1}{T} \sum_{t=0}^{T-1} u(t)$ over the cycle. Then

$$
\begin{aligned}
\bar{x} & =\frac{1}{T} \sum_{t=0}^{T-1} x_{u}(t, x(0))=\frac{1}{T} \sum_{t=1}^{T} x_{u}(t, x(0)) \\
& =\sum_{t=0}^{T-1} \frac{1}{T} f\left(x_{u}(t, x(0)), u(t)\right) \geq f(\bar{x}, \bar{u})
\end{aligned}
$$

by convexity of $f$. Moreover, by convexity of $c$

$$
\bar{c}:=c\left(x_{u}(t, x(0))\right) \geq c(\bar{x}, \bar{u}) .
$$

Now, Lemma 1 implies the existence of a feasible equilibrium $x^{\prime}, u^{\prime}$ improving over the cost of the average state and 
input $c\left(x^{\prime}, u^{\prime}\right) \leq c(\bar{x}, \bar{u})$. Hence $c\left(x^{\prime}, u^{\prime}\right) \leq c(\bar{x}, \bar{u}) \leq \bar{c}$, which is the desired result.

Note that this result does not require Assumption 1 (iv). In a next step, we show that the system is optimally operated at steady-state, that is, that the average infinite horizon cost of any feasible trajectory is lower bounded by the cost in the optimal equilibrium. To do so, let us first introduce the set of all feasible state-action pairs that lead to an (incremental) cost that is less than or equal to the cost incurred in the optimal equilibrium:

$$
\mathbb{Y}_{c^{*}}:=\left\{(x, u) \in \mathbb{X} \times \mathbb{U}: c(x, u) \leq c^{*}\right\}
$$

Also, the following technical lemmas will be useful:

Lemma 2: The set $\mathbb{Y}_{c^{*}}$ is compact.

Lemma 3: For any infinite sequence $c$ with

$$
\bar{c}:=\liminf _{T \rightarrow \infty} \frac{1}{T} \sum_{t=0}^{T-1} c(t)=c^{*}-\delta<c^{*}
$$

and $\delta>0$, there exists a subsequence with indices $T:=$ $\left(t_{1}, t_{2}, t_{3}, \ldots\right)$ such that for all $k \in \mathbb{Z}^{+}$

(i) $c\left(t_{k}\right) \leq c^{*}$ and

(ii) $\frac{1}{t_{k+1}-t_{k}} \sum_{\tau=t_{k}}^{t_{k+1}-1} c(\tau) \leq c^{*}-\frac{\delta}{2}$.

The proofs are provided in the appendix. We are now ready to state the main result:

Theorem 2: CSM systems with CSM objectives satisfying Assumption 1 are optimally operated at steady-state, that is, the minimum average infinite horizon cost achievable is equal to the cost in any optimal equilibrium

$$
c_{\infty}^{*}:=\inf _{x(0), u} \liminf _{T \rightarrow \infty} c\left(x_{u}(t, x(0))\right)=c^{*}
$$

Proof: By definition, $c_{\infty}^{*} \leq c^{*}$. Assume for the sake of contradiction that there exists an initial state $x(0) \in \mathbb{X}$ and a sequence $u(t) \in \mathbb{U}$, such that

$$
\inf _{x(0), u} \liminf _{T \rightarrow \infty} c\left(x_{u}(t, x(0))\right)=c^{*}-\delta<c^{*}
$$

for some $\delta>0$. The proof relies on partitioning the trajectory into suitable segments, such that the system converges towards a cluster point at the end of each of these intervals. Then, a procedure to construct an improving equilibrium from the trajectory during these intervals similar to the proof of Theorem 1 is described.

Consider the sequence $c=(c(x(0), u(0)), c(x(1), u(1), \ldots)$ and let $T:=\left(t_{1}, t_{2}, t_{3}, \ldots\right)$ be a sequence of indices in accordance with Lemma 3. Since $\mathbb{Y}_{c^{*}}$ is compact, the sequence $\left(x\left(t_{k}\right), u\left(t_{k}\right)\right)$ has a cluster point $\left(x_{\infty}, u_{\infty}\right) \in \mathbb{Y}_{c^{*}}$. Therefore, one can define new time indices $\mathbb{S}:=\left\{s_{1}, s_{2}, s_{3}, \ldots\right\} \subseteq T$ such that $x\left(s_{k}\right) \stackrel{k \rightarrow \infty}{\longrightarrow} x_{\infty}$. These new indices induce a partition of the time into segments $\left\{s_{k}, \ldots, s_{k+1}-1\right\}$ and we will study the average state and input over each of these segments:

$$
\begin{aligned}
\bar{x}(k) & :=\frac{1}{s_{k+1}-s_{k}} \sum_{\tau=s_{k}}^{s_{k+1}-1} x(\tau), \\
\bar{u}(k) & :=\frac{1}{s_{k+1}-s_{k}} \sum_{\tau=s_{k}}^{s_{k+1}-1} u(\tau) .
\end{aligned}
$$

Because of convexity of the cost function $c$ and Lemma 3, it follows that

$$
c(\bar{x}(k), \bar{u}(k)) \leq \frac{1}{s_{k+1}-s_{k}} \sum_{\tau=s_{k}}^{s_{k+1}-1} c(x(\tau), u(\tau)) \leq c^{*}-\frac{\delta}{2}
$$

and therefore, $(\bar{x}(k), \bar{u}(k)) \in \mathbb{Y}_{c^{*}}$. Summing over each interval $\left\{s_{k}, \ldots, s_{k+1}-1\right\}$, we find

$$
\begin{aligned}
\bar{x}(k) & =\frac{1}{s_{k+1}-s_{k}} \sum_{\tau=s_{k}}^{s_{k+1}-1} x(\tau) \\
& =\frac{x\left(s_{k}\right)-x\left(s_{k+1}\right)}{s_{k+1}-s_{k}}+\frac{1}{s_{k+1}-s_{k}} \sum_{\tau=s_{k}+1}^{s_{k+1}} x(\tau) \\
& =\frac{x\left(s_{k}\right)-x\left(s_{k+1}\right)}{s_{k+1}-s_{k}}+\frac{1}{s_{k+1}-s_{k}} \sum_{\tau=s_{k}}^{s_{k+1}-1} f(x(\tau), u(\tau)) .
\end{aligned}
$$

Using $\varepsilon_{k}:=\left(x\left(s_{k}\right)-x\left(s_{k+1}\right)\right) /\left(s_{k+1}-s_{k}\right)$ and convexity of the system equations,

$$
\begin{aligned}
\bar{x}(k) & =\varepsilon_{k}+\frac{1}{s_{k+1}-s_{k}} \sum_{\tau=s_{k}}^{s_{k+1}-1} f(x(\tau), u(\tau)) \\
& \geq \varepsilon_{k}+f(\bar{x}(k), \bar{u}(k))
\end{aligned}
$$

Again, since $\mathbb{Y}_{c^{*}}$ is compact and $(\bar{x}(k), \bar{u}(k)) \in \mathbb{Y}_{c^{*}}$, it follows that the sequence $(\bar{x}(k), \bar{u}(k))$ has a cluster point $\left(\bar{x}_{\infty}, \bar{u}_{\infty}\right) \in$ $\mathbb{Y}_{c^{*}}$. Therefore, one can yet again define new indices $K:=$ $\left\{k_{1}, k_{2}, k_{3}, \ldots\right\} \subseteq \mathbb{Z}_{0}^{+}$such that $\bar{x}\left(k_{i}\right) \stackrel{i \rightarrow \infty}{\longrightarrow} \bar{x}_{\infty}$.

Taking the limit as all indices go to infinity we note that $x\left(s_{k}\right) \stackrel{k \rightarrow \infty}{\longrightarrow} x_{\infty}, \bar{x}\left(k_{i}\right) \stackrel{i \rightarrow \infty}{\longrightarrow} \bar{x}_{\infty}$ and $\varepsilon_{k} \stackrel{k \rightarrow \infty}{\longrightarrow} 0$. It follows that $\left(\bar{x}\left(k_{i}\right), \bar{u}\left(k_{i}\right)\right) \stackrel{i \rightarrow \infty}{\longrightarrow}\left(\bar{x}_{\infty}, \bar{u}_{\infty}\right) \in \mathbb{Y}_{c^{*}}$. In the limit, equation (3) turns into $\bar{x}_{\infty} \geq f\left(\bar{x}_{\infty}, \bar{u}_{\infty}\right)$. Now, Lemma 1 implies the existence of an equilibrium $x^{\prime}, u^{\prime}$ improving over the cost $c\left(\bar{x}_{\infty}, \bar{u}_{\infty}\right)$, i.e. $c\left(x^{\prime}, u^{\prime}\right) \leq c\left(\bar{x}_{\infty}, \bar{u}_{\infty}\right)$. Using the bound derived in (2), it follows that

$$
c\left(x^{\prime}, u^{\prime}\right) \leq \lim _{k \rightarrow \infty} c(\bar{x}(k), \bar{u}(k)) \leq c^{*}-\frac{\delta}{2}
$$

which contradicts the initial assumption that $c^{*}$ is the cost incurred in the optimal equilibrium and therefore completes the proof.

In the context of economic MPC [11], [12], [13], [14], systems that are optimally operated at steady-state have been of particular interest and a connection between these systems and dissipative systems has been established: A system (1) is dissipative on a set $\mathbb{X} \times \mathbb{U} \subset \mathbb{R}^{n+m}$ with respect to the supply rate $s: \mathbb{X} \times \mathbb{U} \rightarrow \mathbb{R}$ if there exists a storage function $\lambda: \mathbb{X} \rightarrow \mathbb{R}_{0}^{+}$such that

$$
\lambda(f(x, u))-\lambda(x) \leq s(x, u) \forall(x, u) \in \mathbb{X} \times \mathbb{U} .
$$

In fact, by combining results from [12], [15], [14] it has been shown that under some technical assumptions (closely related to Assumption 1), dissipativity with respect to the supply rate $s(x, u)=c(x, u)-c^{*}$ is a necessary and sufficient condition for optimal steady state operation. From Theorem 2, it therefore immediately follows:

Corollary 1: Let system (1) be a CSM system and $c(x, u)$ a CSM cost, satisfying Assumption 1. Then, system (1) is 
dissipative on $\mathbb{X} \times \mathbb{U}$ with respect to the supply rate $s(x, u)=$ $c(x, u)-c^{*}$.

Before proceeding to an application of our main result, it is worth studying the applicability and limitations of the results. Recall, that in contrast to the general definition of a monotone system, we do not require monotonicity of the system dynamics in the control inputs. On the other hand, the standard definition of monotone systems is only concerned with the system dynamics, but not the objective, whereas we restrict ourselves to control objectives that are monotone in the state. In fact, quadratic objectives of the form $\| x(t)-$ $x^{*} \|_{Q}^{2}$ with $Q \succeq 0$ and $x^{*}$ being the desired steady state are a common choice in control. But if the state is not constrained to $x(t) \geq x^{*}$, such an objective is not monotone in the state and the presented results do not apply as demonstrated by the following counterexample.

Example 1: Consider the system with state $x \in \mathbb{R}_{+}^{2}$ and input $u \in[0,2]$ with system dynamics

$$
x(t+1)=\left(\begin{array}{c}
u \\
x_{1}(t)^{2}
\end{array}\right)
$$

and cost $c(x(t), u(t)):=\sqrt{x_{t}(1)^{2}+\left(x_{t}(2)-1\right)^{2}}$. It is easy to verify that the system dynamics are CSM (in $\mathbb{X} \times \mathbb{U}$ ) and that the cost is convex, but not monotone in the state. We can parametrize all equilibria as $x_{1}=u, x_{2}=u^{2}$ with steady state costs of $\sqrt{u^{2}+\left(1-u^{2}\right)^{2}} \geq \frac{\sqrt{3}}{2}$. But the cycle $x_{u}(t, x(0))$ with $x(0)=\left(\begin{array}{ll}1 & 0\end{array}\right)^{\top}, u(0)=0$ and $u(1)=1$ achieves a better average cost of $c\left(x_{u}(t, x(0))\right)=\frac{1}{2}(\sqrt{1+1}+0)=\frac{\sqrt{2}}{2}<\frac{\sqrt{3}}{2}$. Therefore, this system is optimally operated off steady state. A second counterexample illustrates the role of the state constraints in potentially causing a system to not be optimally operated in steady state.

Example 2: Consider the system with state $x \in \mathbb{R}_{+}^{2}$ and input $u \in[0,4]^{2}$ with system dynamics

$$
x(t+1)=x(t)+\left(\begin{array}{l}
-2+u_{1}(t)^{2} \\
-2+u_{2}(t)^{2}
\end{array}\right),
$$

and cost $c(x(t), u(t)):=\|u(t)\|_{1}$. It is easy to verify that this is a CSM system with a CSM cost. However, note that the set $\mathbb{X}$ is not control invariant and therefore violates Assumption 1. Any equilibrium requires $u_{1}=u_{2}=\sqrt{2}$ with steadystate cost $c(x, u)=2 \cdot \sqrt{2}$. But the cycle $x_{u}(t, x(0))$ with $x(0)=\left(\begin{array}{ll}4 & 0\end{array}\right)^{\top}, u(0)=\left(\begin{array}{ll}0,2\end{array}\right)^{\top}$ and $u(1)=(2,0)^{\top}$ achieves the average cost of $c\left(x_{u}(t, x(0))\right)=\frac{2+2}{2}=2$. Therefore, this system is optimally operated off steady-state. Without state constraints, the cost optimal trajectory makes the system diverge.

\section{Application}

We consider the problem of freeway ramp metering [16], [17], for a single freeway with its dynamics given by the asymmetric, monotone Cell Transmission Model (CTM) [19], [20]. In the CTM, the freeway is partitioned into cells of length $l_{k}$. The state of the mainline is described by the traffic density $\rho_{k}(t)$ of cell $k$ at time $t$, i.e. the number of cars per length in each cell. The density is constrained to
$0 \leq \rho_{k}(t) \leq \bar{\rho}_{k}$, with the traffic jam density $\bar{\rho}_{k}$. The density evolves over time according to the conservation equation

$$
\rho_{k}(t+1)=\rho_{k}(t)+\frac{\Delta t}{l_{k}}\left(\phi_{k-1}(t)+r_{k}(t)-\frac{1}{\bar{\beta}_{k}} \phi_{k}(t)\right) .
$$

Here, the mainline flows are denoted as $\phi_{k}(t)$ and the metering rates as $r_{k}(t)$. The metering rates are equivalent to the inflow to the freeway from the onramps.

The CTM models the flow $\phi_{k}(t)$ as a function of the traffic densities, which is represented by the so-called fundamental diagram, depicted in Figure 1b. The fundamental diagram for each cell can be decomposed in the concave, monotone nondecreasing traffic demand $d_{k}\left(\rho_{k}(t)\right)$, i.e. the number of cars that seek to travel downstream, and the concave, monotone nonincreasing supply of free space $s_{k}\left(\rho_{k}(t)\right)$ in a cell. The flow $\phi_{k}(t)$ is then given as the minimum of upstream demand and downstream supply $\phi_{k}=\min \left\{d_{k}\left(\rho_{k}(t)\right), s_{k+1}\left(\rho_{k+1}(t)\right)\right\}$. The equations are slightly adapted for the first $\phi_{0}(t)=$ $\min \left\{d_{0}, s_{1}\left(\rho_{1}(t)\right)\right\}$ and last cell $\phi_{n}(t)=d_{n}\left(\rho_{n}(t)\right)$. Here, $d_{0}$ is a constant, external traffic demand. We make the assumption of constant split ratios, which means that the outflows from the offramps are modeled as percentages $\beta_{k}$ (often called the split ratios) of the mainline flows at the offramp in cell $k$. For notational convenience, we also use $\bar{\beta}_{k}:=1-\beta_{k}$.

The metering rates $r_{k}(t)$ are obviously limited by the traffic demand $\bar{r}_{k}$ at the respective onramp $r_{k}(t) \leq \bar{r}_{k}$. The external demands $\bar{r}_{k}$ are assumed to be constant, since we are ultimately interested in a (quasi-) steady state setting. We also allow for a lower bound $\underline{r}_{k} \leq r_{k}(t)$ modeling a minimal flow that has to be admitted at all times.

We seek to maximize the (average) demand served, given as a nonnegative, linear combination of the cars admitted to enter the freeway $c^{\top} r(t), c \geq 0$. This is equivalent to minimizing $c(\rho(t), r(t)):=-c^{\top} r(t)$. We note that the Total Travel Distance (TTD), commonly used as a short-term objective in ramp metering, is in fact equivalent to using the demand served as the objective in any equilibrium of the CTM. This is because the equilibrium flows can be expressed in terms of the metering rates as $\phi_{k}=\bar{\beta}_{1 . . k} d_{0}+\sum_{i=1}^{k} \bar{\beta}_{i . . k} r_{k}$, with $\bar{\beta}_{i . . k}:=\prod_{j=i}^{k} \bar{\beta}_{i}$, because of the conservation equations and vice versa. The same holds if the infinite horizon average case is considered, because of the finite storage capacity of the freeway.

It has been shown that for concave demand functions $d_{k}(\cdot)$ and concave supply functions $s_{k}(\cdot)$, finite horizon optimal control problems can be cast as convex optimization problems [20]. It has also been shown that monotonicity of the systems equations plays a role in this [21]. However, this observation also raised the possibility of the existence of a "free-lunch opportunity" in ramp metering for constant boundary conditions, wherein throughput is improved by repeating a congestion-decongestion cycle.

The theory for CSM systems now allows us to refute such a result:

Corollary 2: There does not exist a periodic or aperiodic trajectory that improves infinite horizon average demand satisfaction in the monotone CTM over the optimal equilibrium, 


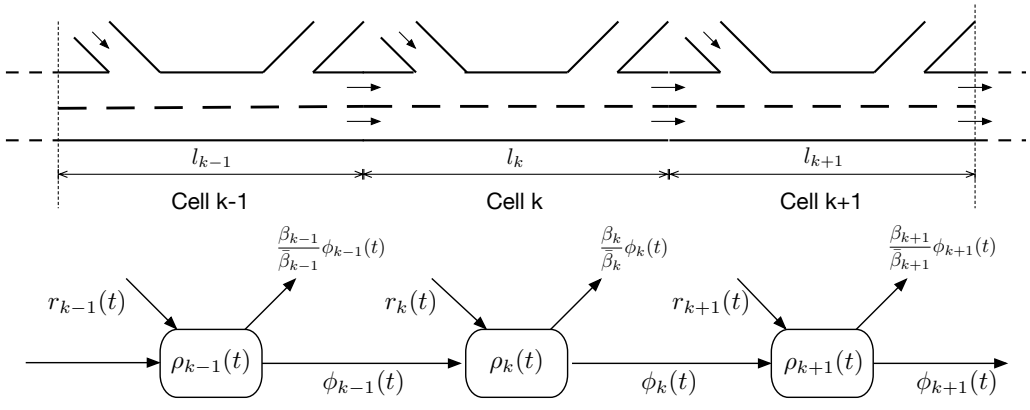

(a) Freeway topology with traffic flows

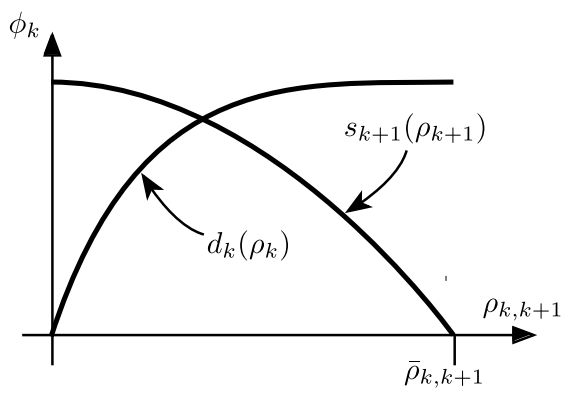

(b) Fundamental diagram

Fig. 1: In the CTM, a freeway is modeled by a combination of conservation equations according to the freeway topology with on- and offramps, as depicted on the left and the fundamental diagram, which characterizes the flow as a function of the densities of upstream and downstream cells, as depicted on the right.

i.e.

$$
\inf _{\rho(0), r} \liminf _{T \rightarrow \infty} c\left(\rho_{r}(t, \rho(0))\right)=\left\{\begin{array}{cl}
\min & c(r, \rho) \\
\text { s.t. } & \rho=f(\rho, r) \\
& \underline{r} \leq r \leq \bar{r}
\end{array}\right.
$$

Proof: Although the CTM in the presented form is monotone, it is easy to verify that it is not convex. Therefore, the results cannot be applied immediately. Instead, we study the following relaxation of the CTM.

We define a system with state $x_{k}(t)$ and controlled inputs $r_{k}(t)$ and $\varphi_{k}(t)$, given by the CSM systems equation

$$
\begin{aligned}
x_{k}(t+1) & =x_{k}(t)+\frac{\Delta t}{l_{k}} . \\
& \left(\varphi_{k-1}(t)+r_{k}(t)+\frac{1}{\bar{\beta}_{k}} \max \left\{-d_{k}\left(x_{k}(t)\right),-\varphi_{k}(t)\right\}\right)
\end{aligned}
$$

with CSM objective

$$
\tilde{c}(x(t), r(t), \varphi(t))=-c^{\top} r(t)+\chi_{x_{k}(t) \leq \bar{\rho}_{k}(t)}+\chi_{\varphi_{k}(t) \leq s_{k+1}\left(x_{k+1}(t)\right)} .
$$

Here, $\chi_{A}$ denotes the characteristic function that is zero, if $x \in A$ and (positive) infinity otherwise. Checking convexity and state monotonicity of the objective is straightforward. For the system equations, note that the demand functions $d_{k}(\cdot)$ is concave, therefore, $-d_{k}(\cdot)$ is convex. Also, the $\max \{\cdot\}$ operator preserves convexity of its arguments. The feasible state set is chosen as $\mathbb{X}:=\mathbb{R}_{+}^{n}$ in accordance with Assumption 1 and the compact, convex input set as $\mathbb{U}:=\left\{u(t)=(r(t), \varphi(t)): \underline{r}_{k} \leq r_{k}(t) \leq \bar{r}_{k}, 0 \leq \varphi_{k}(t) \leq\right.$ $\max \left\{s_{k+1}(0), d_{k}\left(\bar{\rho}_{k}\right)\right\}$. The bounds on $r_{k}(t)$ are taken from the original model, the bounds on $\varphi_{k}(t)$ are bounds on the range of $\phi_{k}(t)$ in the original model and are needed to make the input set $\mathbb{U}$ bounded.

In the following, let $c^{*}$ denote the cost in an optimal equilibrium of the CTM and $c_{\infty}^{*}$ the minimal infinite horizon average cost. We use $\tilde{c}^{*}$ to denote the cost in an optimal equilibrium of the relaxation and $\tilde{c}_{\infty}^{*}$ as the minimal infinite horizon average cost in the relaxation. From Theorem 2, it follows immediately that the relaxed system is optimally operated in steady state. In comparison to the CTM, the controlled inputs $\varphi_{k}$ replace the flows $\phi_{k}$ of the original
CTM and provide us with additional degrees of freedom, but any trajectory of the original CTM can be replicated in the relaxed system with the same costs. Thus, we have that

$$
c_{\infty}^{*} \geq \tilde{c}_{\infty}^{*}=\tilde{c}^{*} .
$$

It remains to be shown that the cost of the optimal steady state of the relaxed system can also be achieved by the CTM. To do so, we construct a suitable equilibrium of the CTM starting from an optimal equilibrium of the relaxation $\left(x^{*}, r^{*}, \varphi^{*}\right)$. Note that for every equilibrium $\min \left\{\varphi_{k}^{*}, d_{k}\left(x_{k}^{*}\right)\right\}=\bar{\beta}_{k} \cdot\left(\varphi_{k-1}^{*}+r_{k}^{*}\right)$. We keep the optimal inflows $r_{k}^{*}$ and propagate the flows through the freeway

$$
\phi_{0}^{*}:=\varphi_{0}^{*}, \phi_{k+1}^{*}:=\bar{\beta}_{k+1}\left(\phi_{k}^{*}+r_{k+1}^{*}\right) \leq \varphi_{k+1}^{*}
$$

By construction, the flows $\phi^{*}$ and metering rates $r^{*}$ satisfy the conservation equations. We then proceed by computing the equilibrium densities as $\rho_{k}^{*}:=d_{k}^{-1}\left(\phi_{k}^{*}\right)$ with the pseudo-inverse of the demand function defined as $d_{k}^{-1}(\phi):=$ $\min \left\{\rho: d_{k}(\rho)=\phi\right\}$. It remains to be checked that $\phi_{k}^{*} \leq$ $s_{k}\left(\rho_{k}^{*}\right)$. Since $\phi_{k}^{*}=d_{k}\left(\rho_{k}^{*}\right) \leq d_{k}\left(x_{k}^{*}\right)$ and by monotonicity of the demand function, it follows that $\rho_{k}^{*} \leq x_{k}^{*}$ and by monotonicity of the supply function $\phi_{k}^{*} \leq s_{k+1}\left(x_{k+1}^{*}\right) \leq s_{k+1}\left(\rho_{k+1}^{*}\right)$, as desired. Therefore, $\left(\rho^{*}, r^{*}\right)$ is indeed an equilibrium of the original CTM, achieving the same cost as the equilibrium of the relaxation, since the metering rates have not been modified. This implies

$$
\tilde{c}^{*}=c^{*}
$$

By definition $c^{*} \leq c_{\infty}^{*}$, and by combining this inequality with equations 4 and 5 , we conclude that $c_{\infty}^{*}=\tilde{c}_{\infty}^{*}=\tilde{c}^{*}=c^{*}$, which completes the proof.

It shall be noted that the construction of a feasible equilibrium starting from the metering rates follows the lines of [21] which provides a detailed characterization of all equilibria of the piecewise-affine CTM. The generalization to the monotone CTM studied here is straightforward.

\section{CONCLUSions}

In this work we have shown that convex, state monotone systems are optimally operated at steady-state. The question about which systems are optimally operated at steady 
state has so far mostly arisen in the context of economic MPC, in which costs are motivated by the actual operating costs/benefits instead of merely being a tuning tool for the control designer. Identification of classes of (nonlinear) systems which are optimally operated at steady state helps to facilitate controller design for the systems that fall into these classes. In particular, any stabilizing controller that makes a convex, state-monotone system converge to an optimal equilibrium is optimal with respect to the average, infinite horizon costs. Such a controller may be much easier to design and to certify than an economic MPC that stabilizes (optimal) cycles.

We applied our theoretical results to a problem in freeway ramp metering, for freeways modeled by the monotone CTM and disproved a conjecture about the existence of a "freelunch opportunity" by operating the system off steady-state. This result serves to reinforce trust in the CTM as a suitable tool for modeling freeway traffic since practical experience seems to contradict the existence of improving cycles for freeway ramp metering.

Based on these results, two main future research directions seem promising: On one hand, efforts should focus on identifying further classes of systems that are optimally operated at steady state. One the other hand, even though the importance of exploiting monotonicity and in particular, the combination of monotonicity and convexity in controller design has been stressed [7], it seems that results in literature are not fully developed yet.

\section{APPENDIX}

Proof: [of Lemma 2] By assumption, $\mathbb{X}$ and $\mathbb{U}$ are closed. Since $c$ is convex and lower semi-continuous, its level sets are closed. The intersection of two closed sets is closed, which implies that $\mathbb{Y}_{c^{*}}$ is closed.

Assumption 1 (i) ensures that any $x \in \mathbb{X}$ is component-wise lower bounded by some $\underline{x}$. Likewise, Assumption 1 (iv) ensures that any $x$ with $(x, u)$ being contained in the level set $\left\{(x, u): c(x, u) \leq c^{*}\right\}$ is component-wise upper bounded by some $\bar{x}$. Since $\mathbb{U}$ is compact, there exist bounds $\underline{u} \leq u \leq \bar{u}$ for all $u \in \mathbb{U}$. Thus, $\mathbb{Y}_{c^{*}} \subseteq\{(x, u): \underline{x} \leq x \leq \bar{x}, \underline{u} \leq u \leq \bar{u}\}$ and it is bounded.

Closedness and boundedness of $\mathbb{Y}_{c^{*}}$ imply compactness.

Proof: [of Lemma 3] It is sufficient to show that for any $t_{k} \in \mathbb{Z}_{0}^{+}$, there exists $t_{k+1}>t_{k}$ that satisfies the conditions (i) and (ii). Assume there does not exist a $t_{k+1}$ satisfying condition (ii). Then $\frac{1}{T-t_{k}} \sum_{\tau=t_{k}}^{T-1} c(\tau)>c^{*}-\frac{\delta}{2} \quad \forall T>t_{k}$, which implies $\liminf _{T \rightarrow \infty} \frac{1}{T-t_{k}} \sum_{\tau=t_{k}}^{T-1} c(\tau)=\liminf _{T \rightarrow \infty} \frac{1}{T} \sum_{\tau=0}^{T-1} c(\tau)>$ $c^{*}-\frac{\delta}{2}$, clearly contradicting the initial assumption. Therefore, there exists some $t_{k+1}^{\prime}>t_{k}$ satisfying condition (ii). If $t_{k+1}^{\prime}$ also happens to satisfy condition (i), we may select $t_{k+1}=t_{k+1}^{\prime}$. Otherwise, let $t_{k+1}=\max \left\{t: t<t_{k+1}^{\prime}, c(t) \leq\right.$ $\left.c^{*}\right\}$. Note that $t_{k+1}$ as defined exists and $t_{k}<t_{k+1}$, since otherwise, $\frac{1}{t_{k+1}^{\prime}-t_{k}} \sum_{\tau=t_{k}}^{t_{k+1}^{\prime}-1} c(\tau)>c^{*}$, contradicting that condition (ii) is satisfied for $t_{k+1}^{\prime}$. The index $t_{k+1}$ satisfies condition (i) by definition and it remains to be shown that it also satisfies condition (ii). We know that $c^{*}-\frac{\delta}{2} \geq$ $\frac{1}{t_{k+1}^{\prime}-t_{k}} \sum_{\tau=t_{k}}^{t_{k+1}^{\prime}-1} c(\tau) \geq \frac{\left(t_{k+1}^{\prime}-t_{k+1}\right) c^{*}+\sum_{\tau=t_{k}}^{t_{k+1}-1} c(\tau)}{t_{k+1}^{\prime}-t_{k}}$ which is equivalent to $\frac{1}{t_{k+1}-t_{k}} \sum_{\tau=t_{k}}^{t_{k+1}-1} c(\tau) \leq c^{*}-\frac{\delta}{2} \cdot \frac{t_{k+1}^{\prime}-t_{k}}{t_{k+1}-t_{k}} \leq c^{*}-\frac{\delta}{2}$ as desired.

\section{REFERENCES}

[1] D. Angeli and E. D. Sontag, "Monotone control systems," IEEE Transactions on Automatic Control, vol. 48, no. 10, pp. 1684-1698, 2003.

[2] M. Hirsch and H. Smith, "Monotone maps: a review," Journal of Difference Equations and Applications, vol. 11, no. 4-5, pp. 379-398, 2005.

[3] M. Hirsch, H. Smith et al., "Monotone dynamical systems," Handbook of differential equations: ordinary differential equations, vol. 2, pp. 239-357, 2005.

[4] A. Rantzer, B. S. Rüffer, and G. Dirr, "Separable lyapunov functions for monotone systems," in 52nd IEEE Conference on Decision and Control. 2013, pp. 4590-4594.

[5] H. Ito, B. S. Rüffer, and A. Rantzer, "Max-and sum-separable lyapunov functions for monotone systems and their level sets," in 53rd IEEE Conference on Decision and Control. 2014, pp. 2371-2377.

[6] G. Dirr, H. Ito, A. Rantzer, and B. Rüffer, "Separable lyapunov functions for monotone systems: constructions and limitations," Discrete Contin. Dyn. Syst. Ser. B, 2015.

[7] A. Rantzer, "Scalable control of positive systems," European Journal of Control, vol. 24, pp. 72-80, 2015.

[8] M. Colombino and R. S. Smith, "Convex characterization of robust stability analysis and control synthesis for positive linear systems," in 53rd IEEE Conference on Decision and Control. 2014, pp. 4379-4384.

[9] M. Colombino and R. Smith, "A convex characterization of robust stability for positive and positively dominated linear systems," 2015.

[10] A. Rantzer and B. Bernhardsson, "Control of convex-monotone systems." in IEEE Conference on Decision and Control, 2014, pp. 23782383.

[11] M. Ellis, H. Durand, and P. D. Christofides, "A tutorial review of economic model predictive control methods," Journal of Process Control, vol. 24, no. 8, pp. 1156-1178, 2014.

[12] D. Angeli, R. Amrit, and J. B. Rawlings, "On average performance and stability of economic model predictive control," IEEE Transactions on Automatic Control, vol. 57, no. 7, pp. 1615-1626, 2012.

[13] L. Grüne and M. Zanon, "Periodic optimal control, dissipativity and mpc," in MTNS 2014, pp. 1804-1807.

[14] M. A. Müller, D. Angeli, and F. Allgöwer, "On necessity and robustness of dissipativity in economic model predictive control," IEEE Transactions on Automatic Control, vol. 60, no. 6, pp. 1671-1676, 2015.

[15] M. A. Müller, L. Grüne, and F. Allgöwer, "On the role of dissipativity in economic model predictive control," IFAC-PapersOnLine, vol. 48, no. 23, pp. 110-116, 2015.

[16] M. Papageorgiou, C. Diakaki, V. Dinopoulou, A. Kotsialos, and Y. Wang, "Review of road traffic control strategies," Proceedings of the IEEE, vol. 91, no. 12, pp. 2043-2067, 2003.

[17] M. Papageorgiou and A. Kotsialos, "Freeway ramp metering: An overview," in IEEE Proceedings on Intelligent Transportation Systems, 2000, pp. 228-239.

[18] M. Schmitt, P. Goulart, A. Georghiou, and J. Lygeros, "Flowmaximizing equilibria of the cell transmission model," in IEEE European Control Conference (ECC), 2015. pp. 2634-2639.

[19] C. F. Daganzo, "The cell transmission model: A dynamic representation of highway traffic consistent with the hydrodynamic theory," Transportation Research Part B: Methodological, vol. 28, no. 4, pp. 269-287, 1994.

[20] G. Gomes and R. Horowitz, "Optimal freeway ramp metering using the asymmetric cell transmission model," Transportation Research Part C: Emerging Technologies, vol. 14, no. 4, pp. 244-262, 2006.

[21] G. Gomes, R. Horowitz, A. A. Kurzhanskiy, P. Varaiya, and J. Kwon, "Behavior of the cell transmission model and effectiveness of ramp metering," Transportation Research Part C: Emerging Technologies, vol. 16, no. 4, pp. 485-513, 2008. 